Phosphorus Research Bulletin Vol. 21 (2007) pp. 9-15

\title{
EFFECTS OF IONIC SURFACTANTS ON THE ADSORPTION OF BOVINE SERUM ALBUMIN TO THE SURFACE OF HYDROXYAPATITE
}

\author{
Emiko Motoki, Satoru Ueno, and Saburo Shimabayashi* \\ (*Corresponding author: saburo@ph.tokushima-u.ac.jp)
}

Faculty of Pharmaceutical Sciences, Division of Physical Pharmacy, The University of Tokushima, Sho-machi 1-78-1, Tokushima 770-8505, Japan

\begin{abstract}
Keywords: Bovine serum albumin, hydroxyapatite, dodecyltrimethylammonium chloride, sodium dodecyl sulfate, denaturation

Abstract: The effects of ionic surfactants, dodecyltrimethylammonium chloride (DTMAC) and sodium dodecyl sulfate (SDS), on the adsorption amount of bovine serum albumin (BSA) onto the surface of hydroxyapatite (HAP) were studied. BSA was bound to HAP owing mainly to electrostatic interaction between opposite charges on HAP and BSA in the absence of an additive. The adsorption amount of BSA on HAP increased with a concentration of added DTMAC by virtue of hydrophobic interaction between hydrocarbon chains of DTMAC bound to BSA and those adsorbed on HAP in the range of a low concentration of DTMAC. The adsorption amount, however, decreased with the concentration in the region of a higher concentration of DTMAC. This is because the expansion/denaturation of BSA was caused after the binding of much amount of DTMAC, resulting in an increase in a molecular occupation area of BSA on the HAP surface. On the other hand, in the presence of SDS, the adsorption amount of BSA on HAP monotonously decreased with a concentration of SDS owing to the strong effect of SDS on the expansion and concomitant denaturation of BSA.
\end{abstract}

(Received January 27, 2007; Accepted March 2, 2007)

\section{INTRODUCTION}

Hydroxyapatite $\left(\mathrm{Ca}_{10}\left(\mathrm{PO}_{4}\right)_{6}(\mathrm{OH})_{2}\right.$, HAP) is a main inorganic component of mammalian hard tissues such as teeth and bones. These hard tissues are composite of proteins, polysaccharides, and HAP, where these are forming an intermolecular complex after mutual interactions between them. ${ }^{1,2}$ Bovine serum albumin (BSA), taken as a model protein in the present study, has been well characterized in its adsorption properties to HAP up to now. Serum albumin is one of serum proteins which play important roles in bone conductivity of mammals. ${ }^{3}$

It is also well-known that BSA is adsorbed by HAP through electrostatic attractive force ${ }^{4-7}$ and that this protein, BSA, is denatured by a surfactant. ${ }^{5}$ In the present paper, the adsorption of BSA by HAP will be discussed in detail in the presence or absence of an ionic surfactant, dodecyl trimethyl ammonium chloride (DTMAC, cationic surfactant) and sodium dodecyl sulfate (SDS, anionic surfactant). After confirming the binding of DTMAC and SDS to BSA as well as to HAP, the adsorption mechanism of BSA to HAP will be discussed here, taking into consideration the effects of the surfactants. The effects are of 2 ways; one is denaturation of BSA and the other is the bridging effect between the BSA and HAP surface through the hydrophobic effect. The effects of SDS and DTMAC will be compared with each other.

\section{MATERIALS AND METHODS}

HAP was a product of Nacalai Tesque Co. (Kyoto, Japan), of which specific surface area was about 50 $\mathrm{m}^{2} / \mathrm{g}$. Its molar ratio of calcium/phosphorus in the bulk was determined as 1.67 mole/mole after chemical analysis. According to the $\mathrm{X}$-ray powder diffraction, it was confirmed that the HAP used in the present work exhibited sharp diffraction peaks at diffraction angles of a typical HAP. BSA was purchased from Sigma Co.(U.S.A.). DTMAC was from Tokyo Chemical Industry Co. (Tokyo). SDS was of the BDH (England), which was purchased from Daiichi Pure Chemicals Co. (Tokyo). These were used without further purification.

It was confirmed that the adsorption equilibrium was attained after a simple stirring for 1 day after preparation of a sample solution mixed with the HAP powder. Adsorption amounts of BSA, DTMAC, and SDS on HAP were calculated from a difference in concentrations before and after the adsorption by the $\operatorname{HAP}\left(0.5 \mathrm{~g} / 20 \mathrm{ml}\right.$ at $\left.30^{\circ} \mathrm{C}\right)$. Concentration of BSA was determined by a UV absorptiometry, while concentrations of DTMAC and SDS were determined by an Epton method after separation of a supernatant from the HAP suspension. Solution $\mathrm{pH}$ at an adsorption equilibrium was 7.4 when a mother 
solution contained $0-50 \mathrm{mM}$ of $\mathrm{NaCl}$, while $6.8-6.9$ when it contained a $50 \mathrm{mM}$ phosphate buffer solution.

These pHs were stable and not fluctuated, because the HAP powder in the solution exhibited a buffer function against $\mathrm{pH}^{4}{ }^{4}$ In the case of the phosphate buffer solution, however, buffer functions both by the solution and by the HAP powder competed for the $\mathrm{pH}$, resulting in decrease from 7.4 to 6.8-6.9.

BSA in these solutions was negatively charged because its isoelectric point (i.e.p.) is $\mathrm{pH} 4.2-4.8 .^{5}$ Therefore, we could expect that BSA might strongly interact with a cationic surfactant such as DTMAC, and even with an anionic surfactant such as SDS as well as with HAP because BSA is an amphoteric polyelectrolyte.

The amounts of DTMAC or SDS bound to BSA were determined at $30^{\circ} \mathrm{C}$ by a dialysis method using a Visking cellophane tubing. The concentrations of DTMAC or SDS were determined by an Epton method using chloroform and methylene blue as the same manner as that in the determination of the adsorption amounts of the surfactants by HAP.

Denaturation of BSA after mixing with an ionic surfactant, DTMAC or SDS, was confirmed by a circular dichroism (CD) spectrum obtained by means of a spectropolarimeter (JASCO J-600).

\section{RESULTS AND DISCUSSION}

\section{(1) Binding of DTMAC and SDS to BSA}

DTMAC and SDS were bound to BSA through 2 mechanisms. One is of electrostatic attractive force and the other is of hydrophobic interaction between hydrophobic groups of BSA and those of DTMAC or SDS.

The binding ratio of DTMAC to BSA monotonously increased with a concentration of DTMAC within the concentration range studied, as shown in FIGURE 1 (A). However, it decreased with a concentration of $\mathrm{NaCl}$ added. This result is suggesting that the binding of DTMAC to BSA is electrostatic, because the binding ratio decreases with an ionic strength prepared by $\mathrm{NaCl}$.

The amount of SDS bound to BSA in water decreased after attaining a maximum at $[\mathrm{SDS}]_{\mathrm{eq}}=$ $7-8 \mathrm{mM}$, as shown with a closed diamond $(\diamond)$ in FIGURE 1 (B). It is reported that critical micellization concentration ( $\mathrm{cmc}$ ) of SDS in water is about $8 \mathrm{mM}$. The SDS micelle formed in a mother solution might be more stable than the SDS aggregate formed along the BSA molecule. Therefore, SDS bound to BSA was moved and/or extracted to the mother solution, resulting in decrease in the binding ratio with a concentration of SDS higher than around $8 \mathrm{mM}$.

Similar phenomena were observed in both $50 \mathrm{mM}$ $\mathrm{NaCl}$ and $50 \mathrm{mM}$ phosphate buffer solutions. That is, the binding ratio decreased after attaining a maximum in each case. The ratios in the presence of $\mathrm{NaCl}$ (open square $(\square)$ ) and phosphate buffer (open triangle $(\triangle)$ ) are higher than those in the absence of added salt (closed diamond $(\diamond)$ )). This fact is showing that the salting-out effect is predominant in contrast to the case of the binding mechanism of DTMAC described above. That is, the hydrophobic interaction (i.e., attractive force) operating between hydrocarbon chain of SDS and hydrophobic area of
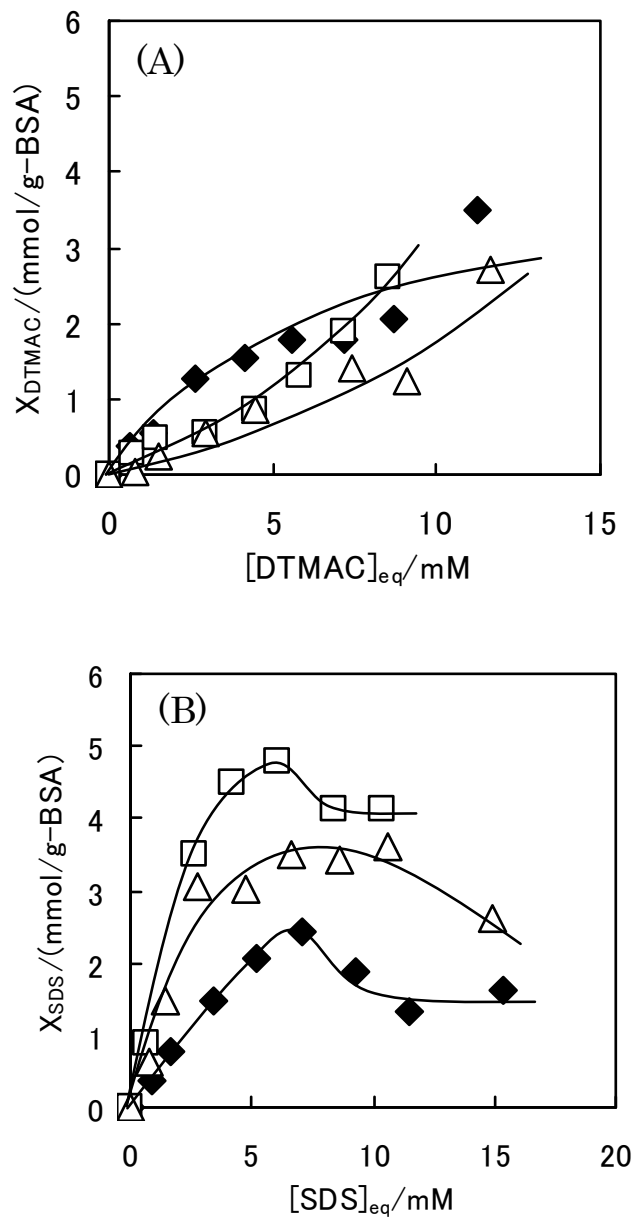

FIGURE 1 Binding isotherms of DTMAC (A) and SDS (B) to BSA as a function of an equilibrium concentration of a surfactant at $30{ }^{\circ} \mathrm{C}$, where an added electrolyte was $50 \mathrm{mM} \mathrm{NaCl}(\square), 50 \mathrm{mM}$ phosphate buffer $(\triangle)$, or none (=just in water, $\diamond$ ). $[\mathrm{BSA}]_{\text {init }}=0.08 \mathrm{w} / \mathrm{v} \% . X_{\mathrm{DTMAC}}$ and $X_{\mathrm{SDS}}$ show the binding amounts of DTMAC and SDS, respectively. 
BSA is more dominant than the electrostatic repulsive force between a dodecyl sulfate anion and a net negative charge of BSA.

\section{(2) Adsorption of DTMAC and SDS on HAP}

Adsorption amounts of DTMAC and SDS by HAP are shown in FIGURE 2. DTMAC was adsorbed on the surface of HAP through electrostatic attractive force between phosphate and hydroxyl anions on HAP and a trimethylammonium cation of DTMAC, while SDS was adsorbed on the surface through electrostatic attractive force between calcium cation on HAP and dodecylsulfate anion of SDS. In addition, SDS was adsorbed on HAP by the mechanism of isomorphorus substitution of sulfate group of SDS for a phosphate ion on the surface. ${ }^{2}$ Therefore, the adsorption amount of SDS by the surface was more than that of DTMAC in the absence of an added electrolyte and in the presence of $50 \mathrm{mM} \mathrm{NaCl}$.

In $50 \mathrm{mM}$ phosphate buffer solution, however, the adsorption amount of DTMAC by HAP was higher than that of SDS. Phosphate ion was preferentially adsorbed on the surface of HAP due to the fact that it is one of the composite ions for HAP. Therefore, the adsorption of DTMAC was accelerated while that of SDS was inhibited by virtue of electrostatic attractive/repulsive force. As a result, the adsorption amount of DTMAC increased while that of SDS decreased in the presence of some amount of phosphate ion, as shown in FIGURE 2.

\section{(3) Effect of added DTMAC on the adsorption amount of BSA to HAP}

Effect of added DTMAC on the adsorption amount of BSA to HAP at a constant concentration of BSA (an initial concentration of BSA, [BSA $]_{\text {init }}=0.2$ w/v\%) is shown in FIGURE 3. At a concentration range of DTMAC lower than $5 \mathrm{mM}$, the adsorption amount of BSA on HAP without an added electrolyte (closed diamond $(\checkmark)$ ) was almost the same as that in an aqueous solution of $50 \mathrm{mM} \mathrm{NaCl}$ (open square ( $\square)$ ). However, the adsorption amount in $50 \mathrm{mM}$ phosphate buffer solution (open triangle $(\triangle)$ ) was remarkably low.

The adsorption amount increased with a concentration of DTMAC over the low concentration region ( $\leqq 5 \mathrm{mM}$ ), as shown in left-hand side in FIGURE 3. The adsorption of BSA on HAP was promoted with a concentration of DTMAC by virtue
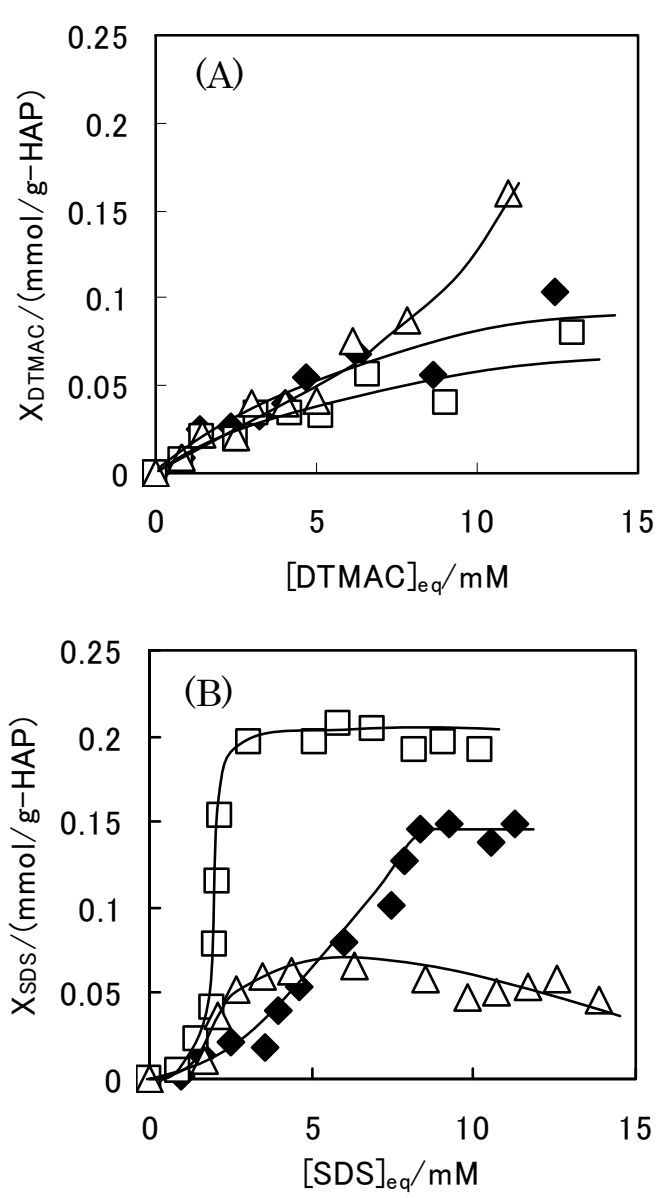

FIGURE 2 Adsorption isotherms for DTMAC (A) and SDS (B) by HAP as a function of an equilibrium concentration of a surfactant at $30^{\circ} \mathrm{C}$, where an added electrolyte was $50 \mathrm{mM} \mathrm{NaCl}$ ( $\square$ ), $50 \mathrm{mM}$ phosphate buffer $(\triangle$ ), or none (=just in water, $)$. Concentration of HAP was kept constant at $0.5 \mathrm{~g} / 20 \mathrm{ml}$. $X_{\text {DTMAC }}$ and $X_{\text {SDS }}$ show the adsorption amounts of DTMAC and SDS, respectively.

of hydrophobic interaction between dodecyl groups of DTMAC bound to BSA and those adsorbed on HAP as well as of hydrophobic interaction between BSA for itself and DTMAC adsorbed by HAP. The binding mechanism is illustrated in SCHEME 1 (b).

In the absence of DTMAC, BSA is adsorbed to the surface of HAP by virtue of electrostatic attractive force between charges on BSA and opposite charges on HAP, as shown in SCHEME 1 (a). Needless to say, the electrostatic attractive force should be still operating even in the presence of DTMAC, as shown in (b), where electric charges are omitted in order to simplify the scheme. 


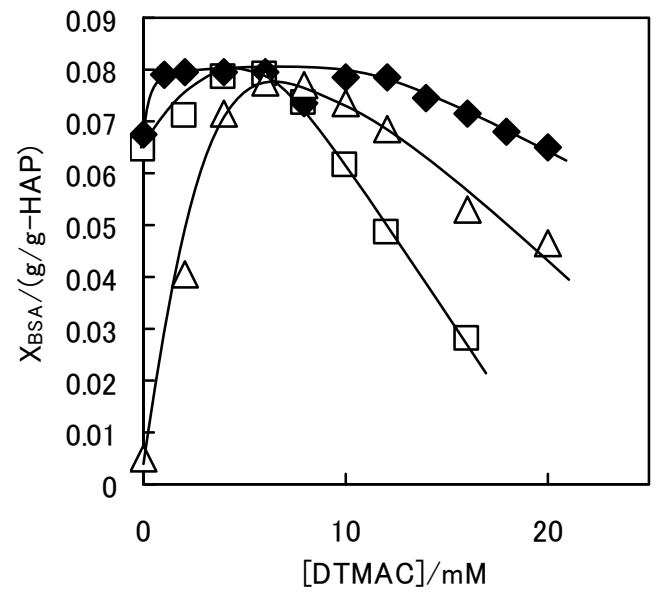

FIGURE 3 Adsorption amounts of BSA on HAP as a function of a concentration of added DTMAC, where an added electrolyte was $50 \mathrm{mM} \mathrm{NaCl}(\mathrm{pH}$ 7.4, $\square$ ), $50 \mathrm{mM}$ phosphate buffer (pH 6.9, $\triangle$ ), or none (=just in water, $\diamond$ ). [HAP] $=0.5 \mathrm{~g} / 20 \mathrm{ml}$. $[B S A]_{\text {init }}=0.2 \mathrm{w} / \mathrm{v} \%$. Data was quoted from a previous paper. ${ }^{9}$ The ordinate shows the adsorption amounts of BSA.

At the domain of the higher concentration of DTMAC, the adsorption amount decreased on the contrary. This should be owing to the expansion/ denaturation of BSA $^{6,7}$ after binding DTMAC, as illustrated in SCHEME 1 (c). ${ }^{8}$

The concentration of DTMAC at a maximum adsorption amount of BSA shown in FIGURE 3 is approximately $5 \mathrm{mM}$ in common. At this concentration of DTMAC, both the binding ratio of DTMAC to BSA (FIGURE 1) and the adsorption amount of DTMAC by HAP (FIGURE 2) seem to roughly attain a half of the saturated amounts. Therefore, we could assume that hydrophobic interaction between DTMAC on BSA and that on HAP might become easiest and most stable at this concentration, because the hydrocarbon chains protruding from BSA and those from HAP penetrate each other easily and form the palisade along the interface between BSA and HAP (see SCHEME 1 (b) and (c)). This effect resulted in attaining a maximum in the adsorption amount of BSA to HAP, where the hydrocarbon chains of DTMAC intervene between BSA and HAP and tightly interconnect with each other.

CD spectra of BSA were obtained in order to monitor the change in its secondary structure after the interaction with DTMAC. FIGURE 4 shows the CD spectra for BSA at various concentrations of DTMAC. The spectra moved upward with a concentration of DTMAC. That is, BSA was denatured by the addition and binding of DTMAC. It spread out its molecular occupation area on the surface of HAP. Therefore, the adsorption amount of BSA on HAP decreased with a concentration of added DTMAC after attaining a maximum at 5-8 mM DTMAC, as shown in FIGURE 3. Schematic illustration for the adsorption mechanism of BSA on HAP is shown in SCHEME 1 (c), where the BSA molecule is sketched elongated in shape in order to depict the denaturation on the surface in the presence of DTMAC. It might be regarded as a kind of surface denaturation. ${ }^{8}$

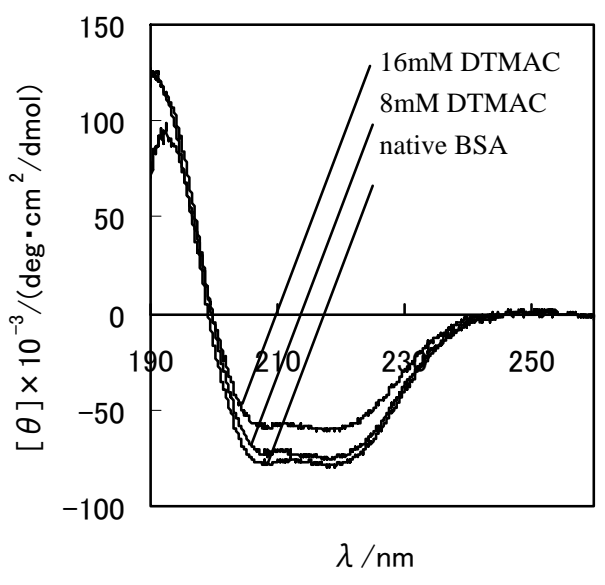

FIGURE 4 Circular dichroism spectra of BSA at a room temperature. The spectra were obtained at 0,8 , and $16 \mathrm{mM}$ DTMAC. [BSA] $=0.016 \mathrm{w} / \mathrm{v} \%$. Data was quoted from a literature. ${ }^{9}$ The spectrum shown with "native BSA" is that of BSA in an aqueous solution without DTMAC. The ordinate shows molar ellipticity of BSA.

The extent of decrease in the adsorption amount at a high concentration of DTMAC in $50 \mathrm{mM} \mathrm{NaCl}$ (open square $(\square)$ ) was larger than that in water (closed diamond $(\diamond)$ ) and that in $50 \mathrm{mM}$ phosphate buffer (open triangle $(\triangle$ ) in FIGURE 3). It was confirmed elsewhere ${ }^{9}$ that denaturation of BSA by 16 mM DTMAC was most accelerated in $50 \mathrm{mM} \mathrm{NaCl}$ when compared among in the presence of $50 \mathrm{mM}$ $\mathrm{NaCl}, 50 \mathrm{mM}$ phosphate buffer, and in the absence of added salt. This sequence of the degree of denaturation is the same as that of the extent of the decrease in FIGURE 3. Therefore, it should be concluded that the adsorption amount of BSA on HAP decreased by the denaturation and expansion.

It was concluded that the addition of a cationic surfactant DTMAC showed two different effects; one is an increasing effect on the adsorption amount of BSA (i.e., promoting the hydrophobic interaction); and the other is a decreasing one on the adsorption amount of BSA (i.e., expansion/ denaturation of BSA 
after the complex formation).

(a) without DTMAC

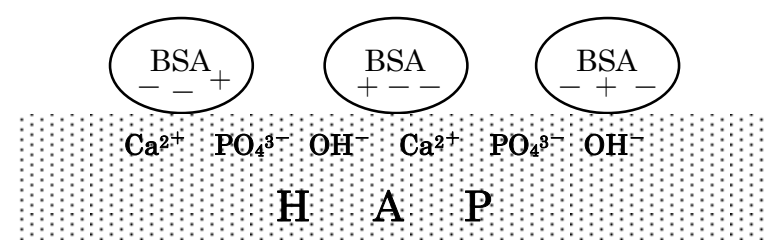

(b) at a low concentration of DTMAC
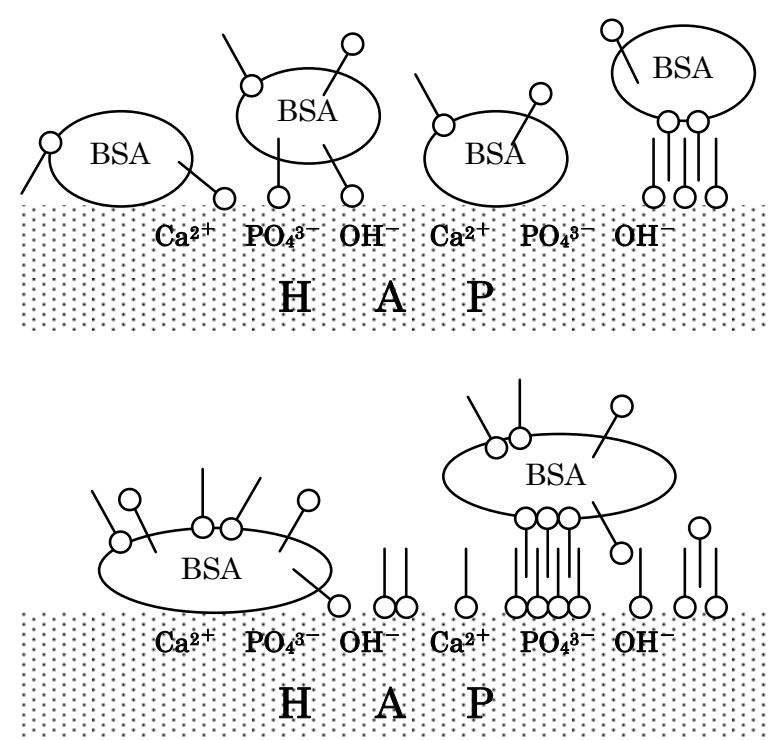

SCHEME 1 Adsorption mechanisms of BSA on the surface of HAP in the presence or absence of DTMAC. BSA is adsorbed on HAP with the side-on conformation to attain the stable binding. BSA in (c) is denatured and expanded after binding much amount of DTMAC.

\section{(4) Effect of added SDS on the adsorption amount of BSA to HAP}

Effect of added SDS on the adsorption amount of BSA to the surface of HAP at a constant concentration of BSA (an initial concentration of BSA, $[B S A]_{\text {init }}=0.2 \mathrm{w} / \mathrm{v} \%$ ) is shown in FIGURE 5 . In water and in an aqueous solution of $50 \mathrm{mM} \mathrm{NaCl}$, the adsorption amount monotonously decreased with a concentration of added SDS. According to the CD spectra, denaturation effect of SDS on BSA was stronger than that of DTMAC, as shown in FIGURE 6. Therefore, BSA molecule expanded and the adsorption amount began to decrease at a low concentration of SDS, as shown in FIGURE 5 (open square $(\square)$ and closed diamond $(\bullet)$ ) and in
SCHEME 2 (b). The effect of promoting the hydrophobic interaction, which was explicitly observed in DTMAC (FIGURE 3 and SCHEME 1), was not observed in SDS. In a region of a higher concentration of SDS, the denaturation was more accelerated and the BSA molecules were more expanded on HAP. Therefore, the adsorption amount more decreased (FIGURE 5). These situations are shown in SCHEME 2 (c).

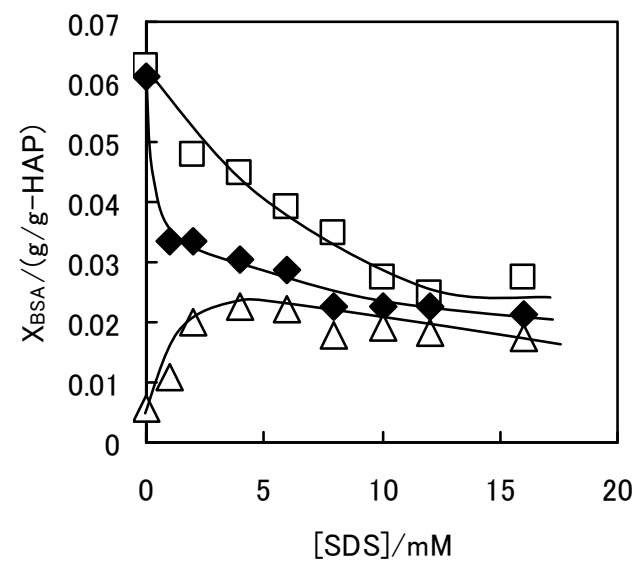

FIGURE 5 Adsorption amounts of BSA on HAP as a function of a concentration of added SDS, where added electrolyte was $50 \mathrm{mM} \mathrm{NaCl}$ (pH7.4, $\square$ ), 50 $\mathrm{mM}$ phosphate buffer (pH6.8, $\triangle$ ), or none (=just in water, $)$. $[\mathrm{HAP}]=0.5 \mathrm{~g} / 20 \mathrm{ml}$. $[\mathrm{BSA}]_{\text {init }}=0.2$ $\mathrm{w} / \mathrm{v} \%$. These concentrations are the same as those used in FIGURE 3. The ordinate shows the adsorption amounts of BSA.

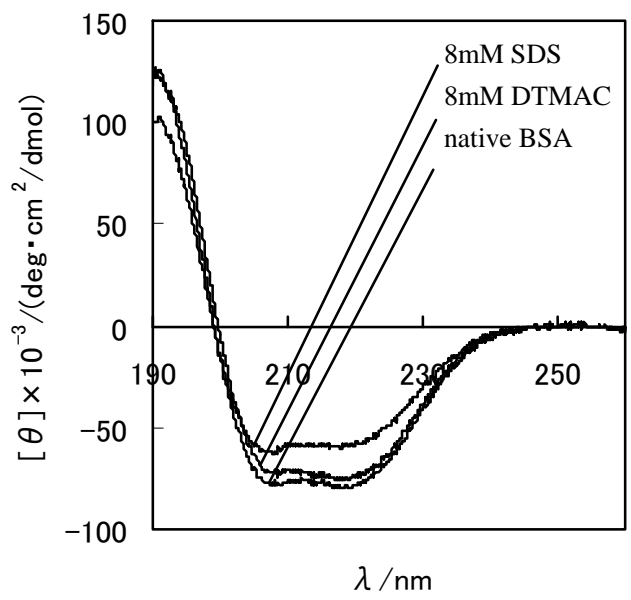

FIGURE 6 Circular dichroism spectra of BSA at a room temperature. The spectra were obtained at 8 mM DTMAC and $8 \mathrm{mM}$ SDS. The spectrum of native BSA was also shown here in order to recognize the effects of these surfactants. The ordinate shows molar ellipticity of BSA. [BSA] = $0.016 \mathrm{w} / \mathrm{v} \%$ 
(a) without SDS

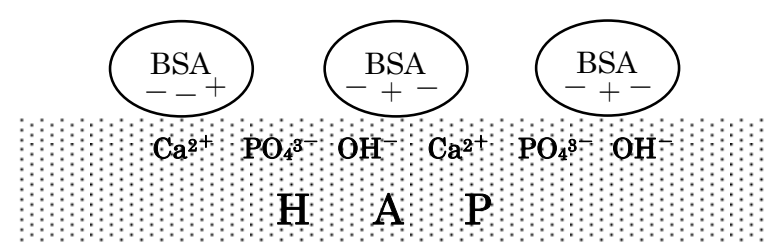

(b) at a low concentration of SDS

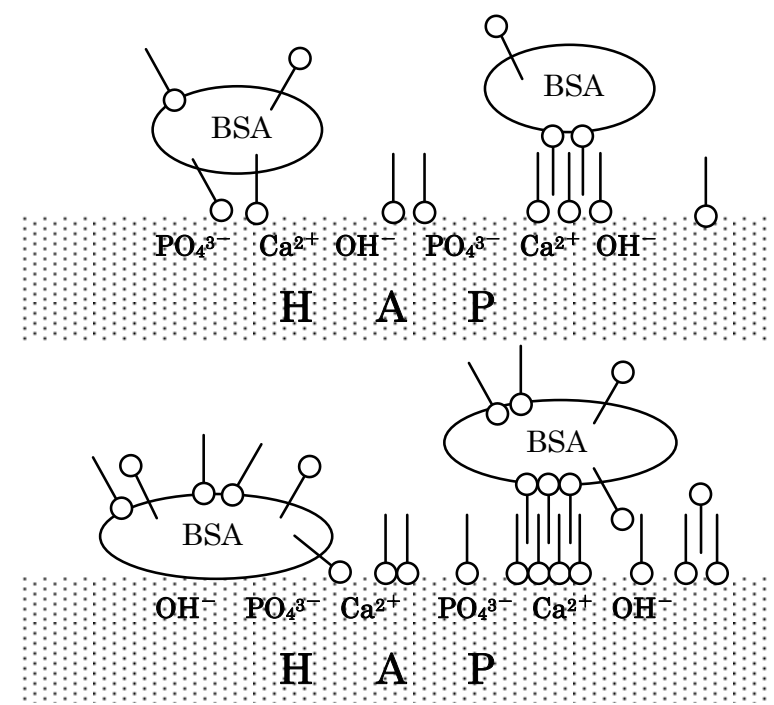

SCHEME 2 Adsorption mechanisms of BSA on the surface of HAP in the presence or absence of SDS. BSA was more denatured in order of (a), (b), and (c), which is schematically shown by elongation of BSA molecules.

The slope of the curve in the presence of $50 \mathrm{mM}$ $\mathrm{NaCl}$ (open square ( $\square$ ) in FIGURE 5) was more gradual than that in water (closed diamond $(\diamond)$ ). According to the results shown in FIGURES 1 and 2, SDS was more bound by both BSA and HAP in 50 $\mathrm{mM} \mathrm{NaCl}$ than in water. This fact suggests that hydrophobic interaction between dodecyl groups of SDS bound to BSA and those adsorbed on HAP could be stronger in the presence of $\mathrm{NaCl}$ than in water. The hydrophobic interaction accelerates the adsorption while the expansion decreases the adsorption amounts in weight. The latter effect is more dominant than the former one as a whole in these SDS systems. However, comparing the systems in the presence and absence of $\mathrm{NaCl}$, the former effect is rather stronger in the presence than in the absence of $\mathrm{NaCl}$. Therefore, the adsorption amount of BSA is higher in the presence than in the absence of $\mathrm{NaCl}$, as shown in FIGURE 5.

In $50 \mathrm{mM}$ phosphate buffer, the adsorption amount of BSA on HAP was very low in the absence of SDS (as shown on the ordinate of FIGURE 5) and in the presence of a low concentration of SDS. This fact is explained in terms of competitive adsorption between phosphate ion and BSA negatively charged. However, the adsorption amount of BSA increased with a concentration of SDS due to the hydrophobic interaction between dodecyl groups of SDS bound to BSA and those adsorbed on HAP. This result is in contrast to that in water and $50 \mathrm{mM} \mathrm{NaCl}$ shown in FIGURE 5, while similar to that shown in FIGURE 3. In a high concentration region of SDS, however, the denaturation effect by SDS mainly appeared and, therefore, the adsorption amount of BSA on HAP decreased.

The effect of SDS on the adsorption amount of BSA to HAP was different from that of DTMAC. The addition of anionic surfactant SDS showed only a decreasing effect on the adsorption amount of BSA both in water and in $50 \mathrm{mM} \mathrm{NaCl}$ (i.e., expansion/denaturation effect of BSA). On the other hand, in $50 \mathrm{mM}$ phosphate buffer, the addition of SDS showed two different effects; one is an increasing effect on the adsorption amount of BSA at a low concentration of SDS and the other a decreasing one on the adsorption amount of BSA at a high concentration of SDS as the same manner as that observed in the presence of DTMAC.

\section{CONCLUSION}

1. DTMAC and SDS were bound to BSA through the electrostatic attractive force and hydrophobic interaction. These surfactants were adsorbed on the surface of HAP through electrostatic attractive force. SDS was also adsorbed in addition by the mechanism of isomorphorus substitution. Therefore, the adsorption amount of SDS on HAP was higher than that of DTMAC.

2. In the region of a low concentration of DTMAC, adsorption amount of BSA to the surface of HAP increased with a concentration of DTMAC due to hydrophobic interaction between dodecyl groups of DTMAC bound to BSA and those adsorbed on HAP. However, after attaining a maximum, the adsorption amount decreased owing to the expansion and denaturation of BSA after binding much amount of DTMAC and increasing the molecular occupation area of BSA.

3. In the case of SDS, the adsorption amount of BSA on HAP monotonously decreased with a concentration of SDS owing to the effect of 
expansion and denaturation of BSA by SDS in water and in the presence of $50 \mathrm{mM} \mathrm{NaCl}$. In 50 $\mathrm{mM}$ phosphate buffer, however, the adsorption amount of BSA decreased after attaining a maximum. The adsorption amount of BSA in a $50 \mathrm{mM}$ phosphate buffer solution was low due to the competitive adsorption of BSA and phosphate ion for the adsorption sites on the HAP surface.

\section{REFERENCES}

1. S. Shimabayashi, K. Kishimoto, and T. Hino, Phosphorus Research Bulletin, 9, 23(2004).

2. S. Shimabayashi and T. Uno, in Calcium Phosphates in Biological and Industrial Systems, Edited by Z. Amjad (Kluwer Academic Publishers, Boston, 1998), Chap. 9, pp. 193-215.

3. M. Nakamura, Phosphorous Letter, 54, 7 (2005).

4. S. Shimabayashi, Y. Kato, and T. Hino, Phosphorus Research Bulletin, 17, 113(2004).

5. K. Aoki, T. Takagi, and H. Terada, Serum Albumin (Kodansha, Tokyo, 1983), p.14.

6. S. Shimabayashi, M. Matsumoto, Phosphorus Research Bulletin, 2, 57(1992).

7. S. Shimabayashi, M. Fukuoka, and T. Hino, Phosphorus Research Bulletin, 18, 65(2005).

8. R. Ishiguro, Y. Yokoyama, H. Maeda, A. Shimamura, K. Kameyama, and K. Hiramatsu, J. Colloid Interface Sci., 290, 91(2005).

9. E. Motoki, S. Ueno, T. Hino, and S. Shimabayashi, Phosphorus Research Bulletin, 20, 135 (2006). 